\title{
Percepção Corporal, Estado Nutricional e Comportamentos de Risco Para os Transtornos Alimentares
}

\author{
Francisco Regis da Silva ${ }^{1}$, Ana Erbênia Pereira Mendes²
}

\begin{abstract}
RESUMO
Objetivou-se com este estudo traçar a imagem corporal, o estado nutricional e os comportamentos de risco para os transtornos alimentares em um grupo de adultos praticantes de musculação em academias de Limoeiro do Norte, Ceará. O estudo tem caráter transversal, do tipo descritivo. Os dados foram coletados em três academias em Limoeiro do Norte, Ceará, domês de agosto de 2013 ao mês de setembro de 2014. A coleta dos dados foi realizada pela aplicação de um protocolo semiestruturado de avaliação nutricional, contendo informações relacionadas à percepção corporal e estado nutricional. Os dados foram tabulados e analisados por meio da estatística descritiva (média, frequências absolutas e relativas, variância e percentual), utilizando o programa Microsoft Excel ${ }^{\circ}$, 2010. A população estudada era constituída predominantemente do sexo masculino, com $72 \%(n=43)$, e $28 \%(n=17)$ do sexo feminino. Em relação à concepção da imagem corporal, $53,48 \%$ $(n=23)$ dos indivíduos do sexo masculino declararam sentir-se bem com sua aparência corporal. Cabe salientar, porém, que $30,23 \%(n=13)$ não estão satisfeitos com sua imagem corporal. No que diz respeito ao sexo feminino, $41,17 \%(n=07)$ se consideravam normal, entretanto $41,17 \%$ ( $n=07)$ não gostam de sua imagem corporal. Já em relação aos sentimentos expressados pelos participantes da pesquisa, ao comerem, além do que consideram suficiente, percebeu-se que $39,53 \%$ ( $n=17$ ) do sexo masculino não sentiam nenhum sentimento com associação a transtornos alimentares, porém $60,46 \%(n=26)$ apresentaram sentimentos, tais como culpa, angústia, chateação e revolta. A população em estudo tem riscos de desenvolver transtornos alimentares, principalmente quando se analisa os sentimentos desenvolvidos em relação "ao ato de comer muito".

Palavras-chave: Transtornos do comportamento alimentar. Imagem corporal. Estado nutricional. Atividade física. Nutrição em saúde pública.

\section{BODY PERCEPTION, NUTRITIONAL STATUS AND RISK BEHAVIORS FOR FOOD DISORDERS}

\section{ABSTRACT}

The objective of this study was to describe body image, nutritional status and risk behaviors for eating disorders in a group of adult bodybuilders in Limoeiro do Norte, Ceará. It is a cross-sectional, descriptive study. The data were collected in three academies located in the city of Limoeiro do Norte, Ceará, during the month of August of 2013 to September of 2014. Data collection was performed through the application of a semi-structured nutritional assessment protocol, containing information related to body perception and nutritional status. Data were tabulated and analyzed using descriptive statistics (mean, absolute and relative frequencies, variance and percentage) using the Microsoft Excel ${ }^{\circledR}$ program, 2010. A predominance of males (SM) was observed with $72 \%(n=43)$; And $28 \%(n=17)$ females (SF). Regarding the conception of the body image, $53.48 \%(n$ $=23$ ) of the individuals of the MS declared to feel normal. However, it should be noted that $30.23 \%(n=13)$ are not satisfied with their body image. With respect to $S F, 41.17 \%(n=07)$ considered themselves normal. However, $41.17 \%(n=07)$ do not like their body image. Regarding the feelings expressed by the participants of the research, in addition to what they considered sufficient, it was observed that $39.53 \%(n=17)$ of MS had no feeling associated with eating disorders, but $60.46 \%(N=26)$ presented feelings, such as: guilty, distressed, upset and disgusted. It can be inferred that the study population has the risk of developing eating disorders, especially when analyzing the feelings developed in relation to "the act of eating a lot".

Keywords: Eating behavior disorders. Body image. Nutritional status. Physical activity. Nutrition in public health.
\end{abstract}

Recebido em: 4/9/2017

Aceito em: $27 / 12 / 2018$

Graduado em Nutrição pelo Instituto Federal de Educação, Ciência e Tecnologia do Ceará - IFCE. Mestre em Saúde Coletiva pelo Programa de Pós-Graduação em Saúde Coletiva da Universidade Estadual do Ceará - UECE. Especialista em Saúde Pública pela Escola de Saúde Pública do Ceará - ESP. Atualmente é Professor Substituto do Curso de Graduação em Medicina da Universidade Estadual do Ceará. http://orcid.org/0000-0001-5470-2874. regisfrs.silva@uece.br

2 Graduação em Nutrição pela Universidade Estadual do Ceará (2008). Mestrado em Ciência e Tecnologia de Alimentos pela Universidade Federal do Ceará (2010). Professora da Universidade Federal do Ceará - Departamento de Economia Doméstica. Tem experiência na área de Nutrição, com ênfase em Avaliação e Controle de Qualidade de Alimentos, Nutrição Funcional e Ciência e Tecnologia de Alimentos. http://lattes.cnpq.br/2097424537744563. erbeniamendes@yahoo.com.br 


\section{INTRODUÇÃO}

A década de 80 foi fortemente marcada pelo aumento progressivo do número de academias de musculação. O fenômeno deve-se ao fato de o corpo ter ganho um espaço marcante na estrutura social vigente, tanto no âmbito da busca por saúde quanto no processo de estereotipagem do corpo (KNOPP, 2008).

Atualmente há uma constante procura pelo melhor condicionamento físico e um forte apelo à forma física com músculos ("corpo malhado"). Isso tem levado pessoas de todas as idades e classes sociais à prática de exercícios em academias. Majoritariamente, os frequentadores são motivados a procurarem as academias por razões estéticas, de saúde ou, ainda, por razões sociais. Seu público frequentador apresenta uma média de faixa etária entre 18 e 35 anos ( $\mathrm{CHI}$ NARELLI; GRAVENA, 2012).

Segundo Pinheiro et al. (2010), nos últimos anos houve uma intensa procura por academias de ginástica, resultante da variedade de atividades oferecidas ao público. A musculação é uma das atividades que está ganhando mais simpatizantes em todo o Brasil. Assim, dados da pesquisa sobre padrões de vida da população brasileira, conduzida no final da década de 90 , indicam que a musculação era a terceira atividade física mais praticada por pessoas acima de 20 anos de idade.

De acordo com Damasceno et al. (2011), atualmente é comum encontrar relatos de pessoas com insatisfação em relação ao seu corpo. Nesta era globalizada, verifica-se uma influência ainda mais forte dos modelos de beleza impostos à população jovem feminina no geral, e, em particular, a masculina. Com efeito, as jovens frequentemente são levadas a utilizar métodos inapropriados para se adequar a um estereótipo corporal predeterminado, difundido por meio da mídia e pela publicidade, que exaltam o sucesso alcançado pelas modelos e atrizes que adotam este padrão. Diante da insatisfação corporal, algumas alternativas são usadas por jovens; as práticas mais comuns são: as dietéticas (caracterizadas pela ingestão calórica insuficiente), os exercícios físicos em excesso, a utilização exacerbada de laxantes, diuréticos e drogas anorexígenas e, até mesmo, o uso de cirurgias plásticas.

A adoção desses comportamentos, visando o "corpo ideal", pode levar ao desenvolvimento de transtornos alimentares, que consistem em patologia de tipo multifatorial, englobando alterações de diversas ordens, como biológica, psíquica e sociocultural. Associado a esses comportamentos, verifica-se carac- terísticas de autoconceito e peso corporal reduzido. Essas preocupações com a aparência podem contribuir para o surgimento de transtornos alimentares, como a anorexia, a vigorexia, a bulimia nervosa, entre outros. Na contemporaneidade, tais transtornos tornaram-se foco relevante de estudo, posto que sua incidência em mulheres jovens pode chegar a até $1 \%$ para anorexia e $4 \%$ quando se trata da bulimia (DAMASCENO et al., 2011).

Para Montenegro, Ornstein e Tapia (2006), há um paradoxo de "corpo perfeito" que se faz presente, definindo dois estereótipos bem distintos: um para o gênero masculino, caracterizado como um corpo musculoso e definido, e outro para o gênero feminino, formado por uma silhueta magra e com baixo percentual de gordura. Para Fracchini (2006), a insatisfação corporal começa na infância, e alguns fatores, como crenças familiares, experiências adversas e fatos socioculturais, influenciam diretamente na concepção de valor de cada indivíduo. Os assuntos mais discutidos no ambiente familiar estão ligados à aparência física e à aceitação social, acarretando em uma série de problemas para a saúde. Adicionalmente, é necessário, para essa população, a ampliação do entendimento sobre saúde, composição corporal e atividade física, de forma que seja promovida a saúde destes sujeitos (FRACCHINI, 2006).

Considerando o cenário apresentado, objetivou-se, com o presente estudo, traçar a imagem corporal, o estado nutricional e os comportamentos de risco para os transtornos alimentares em um grupo de adultos praticantes de musculação em academias de Limoeiro do Norte, Ceará - Brasil.

\section{MÉTODO}

Tratou-se de um estudo com caráter transversal, do tipo descritivo. Os dados foram coletados em três academias localizadas na cidade de Limoeiro do Norte, Ceará, do mês de agosto de 2013 ao mês setembro de 2014, em diferentes turnos.

Realizou-se avaliação quanto à percepção corporal e o estado nutricional, de acordo com o percentual de gordura, e dos comportamentos de risco para o desenvolvimento de transtornos alimentares em um grupo composto por 60 adultos do ambos os sexos. Utilizou-se uma amostra por conveniência.

A coleta dos dados foi realizada por intermédio da aplicação de um protocolo semiestruturado de avaliação nutricional, contendo informações relacionadas à percepção corporal e estado nutricional, adaptado de Kanno (2011). 
As dobras cutâneas tricipitais (DCT), subescapular (DCSE), abdominal (DCAb), suprailíaca (DCSI), axilar média (DCAx), coxa (DCCo) e panturrilha (DCPant), foram mensuradas por meio do adipômetro da marca Sanny, modelo clínico, com graduação de $0,1 \mathrm{~mm}$, com o objetivo de determinar a composição corporal do grupo e o percentual de gordura corporal.

O percentual de gordura corporal (\%GC) foi calculado pela equação de Petroski (1995). As somas das dobras cutâneas foram confrontadas com o padrão de normalidade proposta por Guedes e Guedes (1998).

Em relação à percepção da imagem corporal, essa foi avaliada por meio de um questionário que continha perguntas sobre como os participantes se consideravam, e as respostas eram: magra, normal, se gosta assim e gorda. Já em relação ao sentimento após comer muito, as repostas eram: culpado, chateado, angustiado, revoltado e outros sentimentos. As respostas foram confrontadas com o percentual de gordura corporal investigado. Os dados foram tabulados e analisados por meio da estatística descritiva (média, frequências absolutas e relativas, variância e percentual), utilizando o programa Microsoft Excel $^{\circledR}, 2010$.

Todos os procedimentos empregados neste estudo estão de acordo com determinações institucionais e a Resolução de dezembro n. 466 do Conselho Nacional de Saúde (BRASIL, 2012). O estudo foi submetido e aprovado pelo Comitê de Ética em Pesquisa (Parecer no 888.638) do Instituto Federal de Educação, Ciência e Tecnologia do Ceará - IFCE. Os responsáveis legais dos estabelecimentos em que foram realizadas as coletas assinaram o Termo de Fiel Depositário, autorizando a realização da pesquisa. Além disso, todos os indivíduos foram esclarecidos sobre os procedimentos da coleta antes de assinar o Termo de Consentimento Livre e Esclarecido.

\section{RESULTADOS}

O grupo composto por 60 indivíduos apresentou predominância do sexo masculino (SM) (71,66\% $\mathrm{n}=43$ ). Em relação à idade, o grupo variou de 20 a 59 anos, posto que a maioria apresentou idade entre 20 e 25 anos $(58,14 \%$ - $n=25$ do SM e 53,94\% - n=09 do sexo feminino - SF).

No que diz respeito à concepção da imagem corporal, 53,48\% $(n=23)$ dos indivíduos do SM declararam sentir-se normal. Cabe ressaltar, porém, que $30,23 \%$ ( $n=13$ ) não estão satisfeitos com sua imagem corporal. No que se refere ao SF, obteve-se $41,17 \%$ ( $n=07)$ que se consideravam normal (Gráfico 1).

Das pesquisadas, entretanto, 41,17\% (n=07) não gostam de sua imagem corporal (Gráfico 1), achando-se magra e/ou gorda, quando, na verdade, tais indivíduos não apresentam tal estado nutricional, ou seja, $69,77 \%(n=30)$ e $23,26 \%(n=10)$ do SM apresentaram de leve a moderado percentual de gordura corporal (\%GC), respectivamente. O SF obteve $58,82 \%$ $(n=10)$ e $35,29 \%(n=06)$, leve a moderado \%GC (Gráfico 2).

Gráfico 1 - Concepção da imagem corporal de acordo com a percepção autorreferida, Limoeiro do Norte, CE, 2013-2014

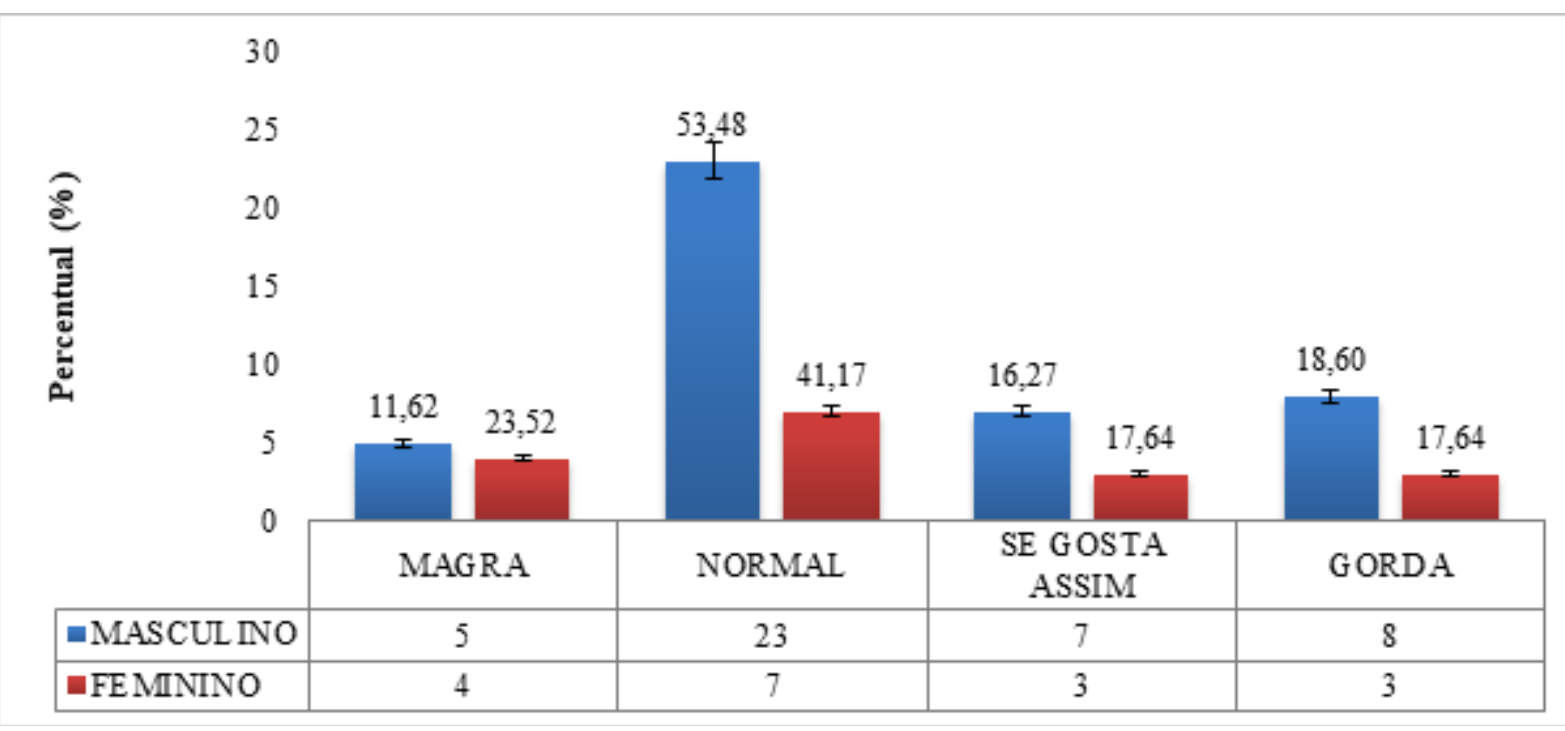

Fonte: Autoria própria (2014). 
Gráfico 2 - Estado nutricional de acordo com o percentual de gordura corporal, Limoeiro do Norte, CE, 2013-2014

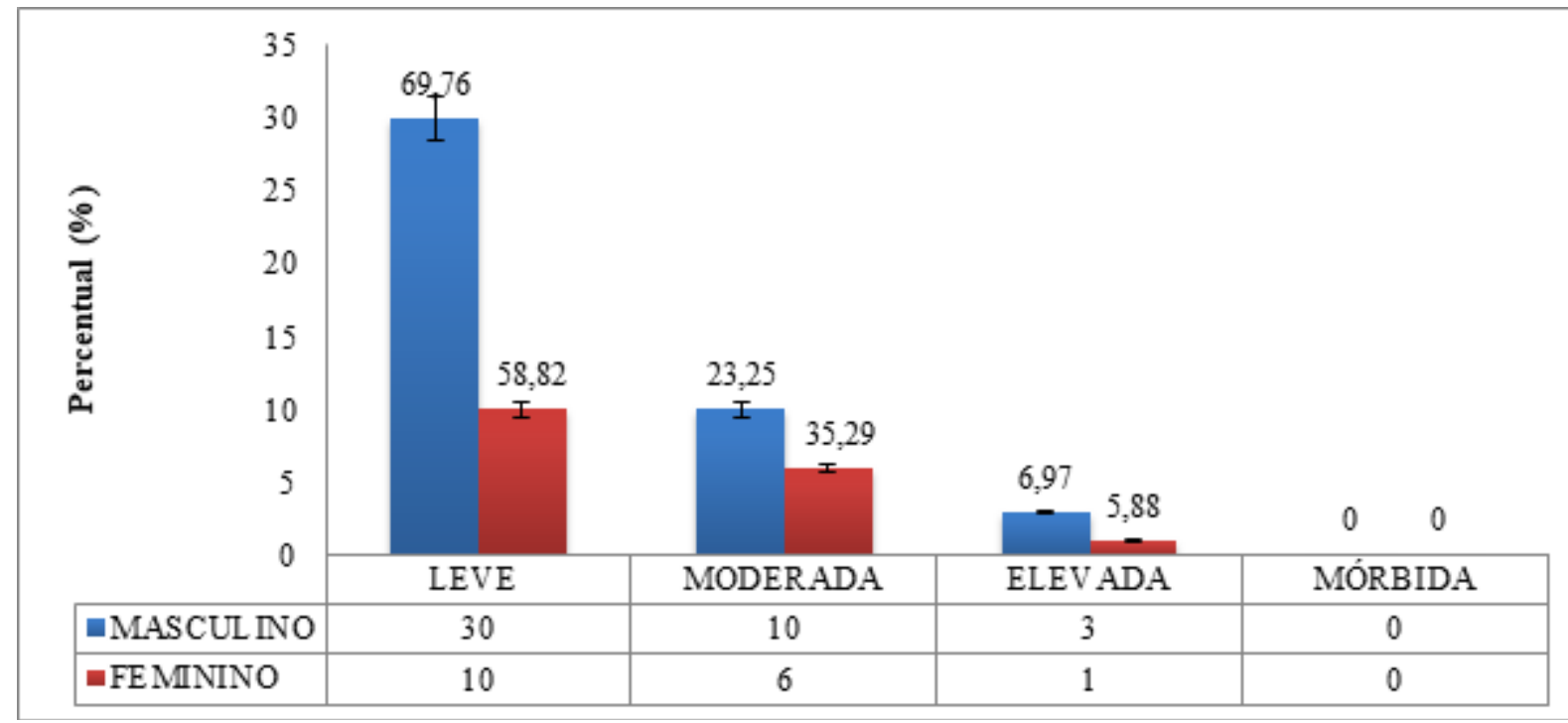

Fonte: Autoria própria (2014).

Gráfico 3 - Concepção da imagem corporal de acordo com a ingestão alimentar, Limoeiro do Norte, CE, 2013-2014

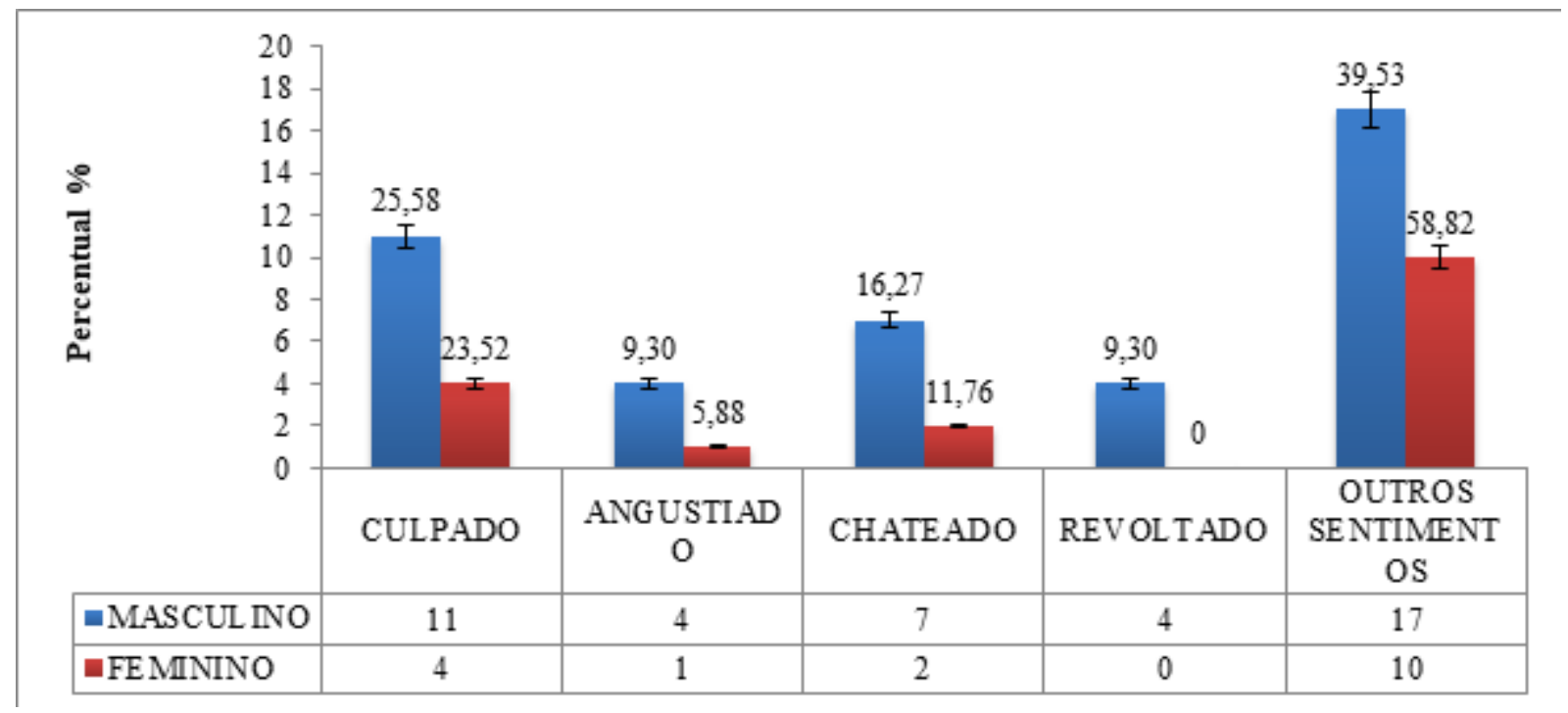

Fonte: Autoria própria (2014).

Já em relação aos sentimentos expressados pelos participantes da pesquisa ao se alimentarem, além do que consideram suficiente, percebeu-se que $39,53 \%(n=17)$ do SM não sentiam nenhum sentimento associado com transtornos alimentares, porém, $60,46 \%(n=26)$ apresentaram sentimentos como: culpado, angustiado, chateado e revoltado. Estes sentimentos apontam para um possível desenvolvimento de transtornos alimentares (Gráfico 3).

\section{DISCUSSÃO}

Fortes, Almeida e Ferreira (2013) avaliaram a possível ocorrência de insatisfação corporal, correlacionando com análise antropométrica, em um gru- po de jovens atletas e sua influência em hábitos alimentares inadequados. No estudo percebeu-se que a insatisfação corporal no SF pareceu ser modulada apenas pelo percentual de gordura (\%GC), ao contrário do SM, em que índice de massa corporal (IMC) e percentual de gordura, juntos, explicaram parte da variância da insatisfação corporal. O comportamento alimentar inadequado no SM foi pouco influenciado pelo percentual de gordura. No SF, tanto o percentual de gordura quanto o IMC não explicaram a variância do comportamento alimentar inadequado, mostrando que, geralmente, a insatisfação corporal tem outros fatores etiológicos envolvidos. A correlação com a an- 
tropometria é um bom indicador, porém ela, por si só, não pode ser um parâmetro de diagnóstico nutricional e médico.

Em um estudo sobre imagem corporal, Fortes e Ferreira (2012) também avaliaram a ocorrência de insatisfação corporal em atletas jovens de diferentes modalidades esportivas; os mesmos averiguaram que, em relação às classificações de insatisfação corporal, quase todos os atletas de ambas as modalidades enquadraram-se na classificação "livre de insatisfação", enquanto apenas dois sujeitos da modalidade futsal foram classificados com "moderada insatisfação". Tal resultado mostra que as modalidades esportivas devem ser uma variável a ser considerada quando se estuda a imagem corporal.

Percebe-se, portanto, que a modalidade esportiva musculação é uma das atividades que mais tem sido procurada por indivíduos propensos a desenvolverem transtornos alimentares diversos. Hausenblas e Downs (2001) realizaram um estudo de meta-análise e encontraram diferenças estatísticas em relação à comparação da imagem corporal de atletas e não atletas, mostrando que os atletas possuem esta mais positiva, e compararam os níveis competitivos, apresentando que atletas de treino considerado de nível médio têm melhor imagem corporal do que os de alto rendimento. Esses mesmos autores identificaram que atletas possuem imagem corporal positiva em relação aos não atletas, porém os atletas estão mais susceptíveis a comportamentos negativos, como excesso de dieta e exercícios (HAUSENBLAS; DOWNS, 2001).

Um estudo com 1.500 estudantes afirmou que os homens são mais satisfeitos que as mulheres em relação à percepção da autoimagem corporal e à prática de exercícios físicos. Os sujeitos chegam a ficar, em média, 2 horas por dia fazendo atividade física para melhorar a aparência, e, apesar de buscarem um corpo diferente do que têm, os homens não se preocupam tanto em cuidar da aparência fora da academia, em comparação com as mulheres (KORN et al., 2013).

De acordo com Pope et al. (1999), existem pessoas que são obsessivas pela sua imagem corporal, mais especificamente pelo volume de seus músculos e de sua definição, ou seja, um corpo com grande volume muscular e quase sem gordura.

Para Smolak, Levine e Thompson (2001), a imagem corporal é um fenômeno que envolve fortemente os gêneros. Antigamente havia o predomínio da ideia de que meninos não apresentavam problemas relacionados à insatisfação corporal. Atualmente, sabe-se que isso não é verdade, pois já são encontrados trabalhos relatando jovens meninos com transtornos alimentares (TEIXEIRA et al., 2009; SCHAAL et al., 2011). Os estudos demonstram, no entanto, que as meninas são mais preocupadas com o peso corporal (SMOLAK; LEVINE; THOMPSON, 2001), e os meninos em aumentar o tamanho e músculos (MCCABE; RICCIARDELLI, 2004).

Vilhena et al. (2012), ao avaliarem a imagem corporal de professores de Educação Física, verificaram que 166 (92,7\%) dos professores e 51 (67,2\%) das professoras não apresentaram distorção de imagem corporal; 13 (7,3\%) da amostra masculina e 25 (32,8\%) da amostra feminina, contudo, apresentaram de leve à moderada distorção de imagem corporal.

Pesquisas sobre a insatisfação corporal feminina (SARDINHA; OLIVEIRA; ARAÚJO, 2008) sugerem que o conflito entre o corpo atual (real) e o corpo desejado parece estimular a adoção de dietas, e que, quando mantidas por períodos prolongados, pode culminar em distúrbios psiquiátricos, como os transtornos alimentares (OLIVA; FAGUNDES, 2001; HAY, 2002).

A cultura do corpo magro afeta, principalmente, a população geral (THOMPSON; CHARD, 2002). Atleta magro, com desenvolvimento musculoesquelético, é uma exigência para a maioria dos esportes; por isso existe uma cobrança por determinados padrões morfológicos para se atingir um desempenho desejado no treinamento esportivo, o que causa uma ligeira discrepância entre o corpo real e o corpo ideal no contexto competitivo (VIEIRA et al., 2009), levando o indivíduo a uma enorme insatisfação com a sua imagem corporal. Nesse sentido, meninos são incentivados a praticar atividades esportivas, enquanto meninas são estimuladas a praticar atividades que promovam o emagrecimento.

\section{CONCLUSÃO}

Diante dos resultados, pode-se inferir que a população em estudo apresenta riscos de desenvolver transtornos alimentares, principalmente quando se analisa os sentimentos desenvolvidos em relação "ao ato de comer em demasia" e pelo baixo percentual de gordura. Vale destacar que a busca pelo corpo, dito "perfeito", pode ser um dos fatores que os levam a autodeclararem tais sentimentos em relação à comida que "não deveria" ser ingerida.

Sugere-se, portanto, estudos que investiguem outros fatores etiológicos que possam influenciar no desenvolvimento dos transtornos alimentares do grupo em estudo, assim como atividades de orientação nutricional e promoção da saúde destes sujeitos. 


\section{REFERÊNCIAS}

BRASIL. Conselho Nacional de Saúde. Resolução no 466, de 12 de dezembro de 2012. Brasília, 2012. Disponível em: http://www.conselho.saude.gov.br/web_comissoes/conep/index.html. Acesso em: 7 jul. 2017.

CHINARELLI, J. T.; GRAVENA, A. F. Insatisfação corporal e comportamento alimentar em frequentadores de academia. Revista Saúde e Pesquisa, v. 5, n. 2, p. 280-287, 2012. Disponível em: http://periodicos.unicesumar.edu.br/index.php/ saudpesq/article/view/2122/1674. Acesso em: 20 jun. 2017.

DAMASCENO, M. L. et al. Associação entre comportamento alimentar, imagem corporal e esquemas de gênero do autoconceito de universitários praticantes de atividade físicas. Revista Brasileira de Atividade Física \& Saúde, v. 16, n. 2, p. 138-143, 2011. Disponível em: https://periodicos.ufpel. edu.br/ojs2/index.php/RBAFS/article/viewFile/572/572. Acesso em: 22 jun. 2017.

FACCHINI, M. La preocupación por el peso y la figura corporal en las niñas y adolescentes actuales: ¿de dónde proviene? Archivos Argentinos Pediatria, v. 104, n. 4, p. 345-350, 2006. Disponible en: http://www.scielo.org.ar/pdf/aap/ v104n4/v104n4a12.pdf. Acceso en: 28 jun. 2017.

FORTES, L. S.; FERREIRA, M. E. C. Satisfação com a imagem corporal e suas relações com variáveis antropométricas em jovens atletas masculinos. Revista Mackenzie de Educação Física e Esporte, v. 11, n. 2, p. 68-81, 2012. Disponível em: http://editorarevistas.mackenzie.br/index.php/remef/article/view/3499/3790. Acesso em: 24 jun. 2017.

FORTES, L. S.; ALMEIDA, S. S.; FERREIRA, M. E. C. Indicadores antropométricos de insatisfação corporal e de comportamentos alimentares inadequados em jovens atletas. Revista Brasileira de Medicina Esportiva, v. 19, n. 1, p. 3539, 2013. Disponível em: http://www.scielo.br/pdf/rbme/ v19n1/07.pdf. Acesso em: 24 jun. 2017.

GUEDES, D. P.; GUEDES, J. E. R. P. Controle de peso corporal: composição corporal, atividade física e nutrição. 1. ed. Londrina: Midiograf, 1998.

HAUSENBLAS, H. A.; DOWNS, D. S. Comparison of body image between athletes and nonathletes - a meta-analytic review. Journal Applied Sports Psychology, v. 13, n. 1, p. 323-339, 2001. Available from: http://www.tandfonline. com/doi/abs/10.1080/104132001753144437?journalCode=uasp20. Cited: 22 jun. 2017.

HAY, P. J. Epidemiologia dos transtornos alimentares: estado atual e desenvolvimentos futuros. Revista Brasileira de Psiquiatria, v. 24, n. 3, p. 13-17, 2002. Disponível em: http://www.scielo.br/pdf/\%0D/rbp/v24s3/13965.pdf. Acesso em: 21 jun. 2017.

KANNO, A. M. Percepção dos profissionais de nutrição quanto ao uso de suplementos alimentares pelos praticantes de musculação. 2011. Monografia (Especialização em prescrição de exercícios físicos na academia) - Instituto de Ciências da Saúde, Universidade Feevale, Novo Hamburgo, RS, 2011.

KORN, L. et al. Health perceptions, self and body image, physical activity and nutrition among undergraduate students in Israel. PLoS One, v. 8, n. 3, p. 1-7, 2013. Available from:: https://www. ncbi.nlm.nih.gov/pmc/articles/PMC3597731/. Cited: 22 jun. 2017.

KNOPP, G. C. A influência da mídia e da indústria da beleza na cultura de corpolatria e na moral da aparência na sociedade contemporânea. In: ENECULT - ENCONTRO DE ESTUDOS MULTIDISCIPLINARES EM CULTURA, 4., 2008, Salvador. Anais [...]. Salvador: Ufba, 2008. p. 1-13. Disponível em: http://www.cult. ufba.br/enecult2008/14415.pdf. Acesso em: 22 jun. 2017.
MCCABE, M. P.; RICCIARDELLI, L. A. A longitudinal study of pubertal timing and extreme body change behaviors among adolescent boys and girls. Adolescence, v. 39, n. 1, p. 145166, 2004. Available from: https://www.researchgate.net/ publication/8478675_A_longitudinal_study_of_pubertal_ timing and extreme body change behaviors among adolescent_boys_and_girls. Cited: 23 jun. 2017.

MONTENEGRO, M. M. A.; ORNSTEIN L. C.; TAPIA, I. P. A. Cuerpo y corporalidad desde el vivenciar femenino. Acta bioethic, v. 12 , n. 2 , p. 165-168, 2006. Disponible en: http://www.scielo.cl/pdf/abioeth/v12n2/art04.pdf. Acceso en: 23 jun. 2017.

OLIVA, C. A. G.; FAGUNDES, U. Aspectos clínicos e nutricionais dos transtornos alimentares. Psiquiatria na Prática Médica, v. 34, n. 2, p. 47-53, 2001. Disponível em: http://www2.unifesp. br/dpsiq/polbr/ppm/atu1_06.htm. Acesso em: 20 jun. 2017.

PETROSKI, E. L. Desenvolvimento e validação de equações generalizadas para a estimativa da densidade corporal em adultos. 1995. Tese (Doutorado em Educação Física) - Universidade Federal de Santa Maria, Santa Maria, RS, 1995.

PINHEIRO, K. C. et al. Barreiras percebidas para prática de musculação em adultos desistentes da modalidade. Revista Brasileira de Atividade Física \& Saúde, v. 15, n. 3, p. 157-162, 2010. Disponível em: https://periodicos.ufpel.edu.br/ojs2/index. php/RBAFS/article/view/716/723. Acesso em: 23 jun. 2017.

POPE, H. G. et al. Envolving ideals ofmale body image as seen through action toys. Int J Eat Disord, v. 26. n. 1, p. 6572, 1999. Available from: https://www.ncbi.nlm.nih.gov/ pubmed/10349585. Cited: 23 jun. 2017.

SARDINHA, A.; OLIVEIRA, A. J.; ARAÚJO, C. G. S. Dismorfia muscular: análise comparativa entre um critério diagnóstico e um instrumento psicológico. Revista Brasileira de Ciências do Esporte, v. 14, n. 4, p. 387-392, 2008. Disponível em: http://www.scielo.br/pdf/rbme/v14n4/v14n4a13.pdf. Acesso em: 22 jun. 2017.

SCHAAL, K. et al. Psychological balance in high level athletes: gender-based differences and sport-specific patterns. Psychopathology and High Level Sport, v. 6, n. 5, p. 1-9, 2011. Available from: https://www.ncbi.nlm.nih.gov/ pubmed/21573222. Cited: 22 jun. 2017.

SMOLAK, L.; LEVINE, M.; THOMPSON, J. K. The use of the Sociocultural Attitudes Towards Appearance Questionnaire with middle school boys and girls. International Journal of Eating Disorders, v. 29, n. 1, p. 216-223, 2001. Available from: https:// www.ncbi.nlm.nih.gov/pubmed/11429984. Cited: 22 jun. 2017.

TEIXEIRA, P. C. et al. A prática de exercícios físicos em pacientes com transtornos alimentares. Revista de Psiquiatria Clínica, v. 36, n. 4, p. 14-152, 2009. Disponível em: http://www.scielo. $\mathrm{br} / \mathrm{pdf} / \mathrm{rpc} / \mathrm{v36n} 4 / \mathrm{a} 04 v 36 n 4 . p d f$. Acesso em: 21 jun. 2017.

THOMPSON, A. M.; CHARD, K. E. The relationship of social physique anxiety to risk for developing and eating disorder in young females. Journal of Adolescence Health, v. 31, n. 2, p. 183-189, 2002. Available from: https://www.ncbi.nlm. nih.gov/pubmed/12127389. Cited: 22 jun. 2017.

VIEIRA, J. L. L. et al. Distúrbios de atitudes alimentares e distorção da imagem corporal no contexto competitivo da ginástica rítmica. Revista Brasileira de Medicina do Esporte, v. 15, n. 6, p. 410-414, 2009. Disponível em: http://www.scielo. br/pdf/rbme/v15n6/a01v15n6.pdf. Acesso em: 22 jun. 2017. VILHENA, L. M. et al. Avaliação da imagem corporal em professores de Educação Física atuantes no fitness na cidade do Rio de Janeiro. Revista Brasileira de Ciências do Esporte, v. 34, n. 2, p. 449-464, 2012. Disponível em: http://www.scielo. br/pdf/rbce/v34n2/a14v34n2.pdf. Acesso em: 22 jun. 2017. 